I N S T I T U T O

DE

M E D I C I N A

T R O P I C A L

$\mathrm{DE}$

SÃO PAULO

JOURNAL OF THE SÃO PAULO INSTITUTE OF TROPICAL MEDICINE

'Universidade de São Paulo, Faculdade de Medicina de Ribeirão Preto, Centro de Cardiologia, Ribeirão Preto, São Paulo, Brazil

Correspondence to: Minna Moreira Dias Romano

Universidade de São Paulo, Faculdade de Medicina de Ribeirão Preto, Centro de Cardiologia, Avenidas dos Bandeirantes, 3900, CEP 14.049-900, Ribeirão Preto, SP, Brazil

E-mail: minna@fmrp.usp.br

Received: 23 March 2021

Accepted: 23 June 2021

\section{Prospective study of ventricular function and myocardial deformation related to survival in acute Chagas disease: an experimental animal model}

\author{
Arthur Lauand Vargas', Beatriz de Paula Dias ${ }^{\circledR 1}$, Henrique Turin Moreira', \\ Edgard Camilo de Oliveira-Filho', Denise Mayumi Tanaka', Marcus Vinicius \\ Simões ${ }^{1}$, Benedito Carlos Maciel ${ }^{1}$, André Schmidt ${ }^{\circledR}$, José Antônio \\ Marin-Neto ${ }^{1}$, Minna Moreira Dias Romano 1
}

\section{ABSTRACT}

Chagas disease (CD) has been changing from an endemic Latino-American disease to a condition found outside endemic regions, due to migratory movements. Although often subclinical, its acute phase can be lethal. This study aimed to assess survival during the acute phase of $\mathrm{CD}$ and its relationship with ventricular function in an experimental model. To this end, 30 Syrian hamsters were inoculated with Trypanosoma cruzi (IG) and other 15 animals received saline solution (CG). Groups were monitored daily and submitted to echocardiography in two moments: before the challenge and 15 days post-infection. Left ventricular ejection fraction (LVEF) and global longitudinal myocardial strain (GLS) of the LV were measured. The IG was divided into groups of animals with and without clinical signs of disease. ANOVA for mixed models was used to compare ventricular function parameters. Survival analysis was studied using Kaplan-Meier curves and the log-rank test. The follow-up lasted 60 days. LVEF in IG was reduced through time (53.80 to 43.55\%) compared to CG (57.86 to $59.73 \%)(\mathrm{p}=0.002)$. There was also a reduction of GLS $(-18.97 \%$ to $-12.44 \%)$ in the IG compared to CG ( $\mathrm{p}=0.012)$. Twelve animals from IG died compared to one animal from CG. Eleven out of the 12 animals from the IG group died before presenting with clinical signs of infection. Survival was reduced in the IG compared to CG over time $(p=0.02)$. The reduced survival during the acute phase of this experimental model of Chagas disease was related to the significant reduction of LV function. The mortality rate in the IG was higher in the group presenting with clinical signs of infection.

KEYWORDS: Echocardiography. Acute Chagas disease. Speckle-tracking. Animal experimental model. Ventricular function. Myocardial deformation.

\section{INTRODUCTION}

Chagas disease (CD) is endemic in Latin America, with more than 8 million people infected by the protozoan Trypanosoma cruzi $^{1-3}$. Migration has spread disease to Europe and North America, with an increased number of cases in the last decade ${ }^{4,5}$. Although vector transmission rates were reduced in endemic regions, non-vector oral transmission or secondary to transfusion and solid organ transplantation are still prevalent ${ }^{6}$. Reactivation of disease in scenarios of immunosuppression, as happens in heart transplant patients, is a particular and dangerous situation ${ }^{7,8}$.

In Brazil, acute $\mathrm{CD}$ is the main infectious disease impacting patients' quality of life, overcoming the Dengue virus infection ${ }^{1}$. The acute phase of CD is mostly 
asymptomatic, but it can be lethal for a proportion of patients, probably secondary to acute myocarditis ${ }^{9,10}$. Among children and young adults who develop symptoms, ventricular dysfunction is not always identified but defines a poor outcome when is present ${ }^{9}$. Although human studies of cases series have reported myocarditis and high mortality rates in the acute phase of $\mathrm{CD}^{11}$, some animal explorations failed to show consistent ventricular dysfunction ${ }^{12}$.

As prospective studies in humans are limited by the characteristics of Chagas disease, animal models became useful to explore evolutionary aspects of cardiac function with great resemblance to human disease ${ }^{13}$. The Syrian hamster model has been described to mimic all phases of Chagas disease with a searchable timeline, early myocardial lesion detection and progression to chronic cardiomyopathy ${ }^{14,15}$. Echocardiography is an accurate and non-invasive tool for the investigation of Chagas cardiomyopathy, assessing left ventricle (LV) geometry and function through the evolution of $\mathrm{CD}^{16}$. New developments such as dedicated small animal equipment and highresolution transducers ${ }^{17}$, in addition to new techniques like Speckle Tracking Echocardiography (STE), have enhanced the ability to study early myocardial damage in $\mathrm{CD}^{18-20}$.

This study aimed to prospectively evaluate LV geometry and myocardial function and their relationships with survival in the acute phase of an animal model of CD. Specific objectives were [1] to assess survival during the acute phase of $\mathrm{CD}$ and its association with LV impairment; [2] to associate the clinical presentation of the animals in the acute phase with the outcome death; [3] to evaluate if STE parameters have prognostic value.

\section{MATERIALS AND METHODS}

\section{Experimental animal model}

Forty-five 12-week old female Syrian hamsters (Mesocricetus auratus) were kept in a climate-controlled environment with free access to water and standard chow under a twelve-hour light/dark cycle. Animals were submitted to all experimental procedures under anesthesia with Ketamine $(100 \mathrm{mg} / \mathrm{kg})$ and Xylazine $(10 \mathrm{mg} / \mathrm{kg})$ to avoid pain. The study and all experimental procedures were approved by the Institutional Animal Research Ethics Committee (protocol No 223/2018).

\section{Experimental protocol}

Animals $(n=45)$ were randomly separated into two groups: the control group (CG) composed of 15 animals injected with saline solution and the infected group (IG) with 30 animals. The infected group was intraperitoneally inoculated with 35,000 trypomastigote forms of Trypanosoma cruzi (Y strain) and had the infection confirmed by a parasite DNA detection in a blood sample by Polymerase Chain Reaction (PCR). The control group was injected with an equal volume of saline solution $(0.2 \mathrm{~mL})$. Animals were daily monitored and submitted to echocardiography in two different moments: before the challenge (baseline) and 15 days post-infection. Clinical signs of acute Chagas disease were characterized by at least one of the following: weight loss, mucous wounds, fur loss or lethargy. The ollow-up lasted 60 days and the survivors rate and time to death were counted.

\section{Echocardiography}

Examinations were performed with the equipment Vevo2100 (Fujifilm Inc., Japan) ultrasound system provided with a 30-MHz high-resolution transducer that is dedicated to small animals Parasternal long and short axis view were obtained. Left ventricular end-diastolic diameter (LVEDD) and left ventricular end-systolic diameter (LVESD) were obtained at the maximum ventricular diastolic dimension, and LVESD at the maximum inward motion of the septum and posterior wall, respectively. The left ventricular mass (LV mass) was estimated by the Devereux formula using thickness measurements of the septum and posterior wall. LVEDD and LV mass were also presented as indexed measures when divided by the animal weight. The LV fractional shortening (FS) was calculated from LVEDD and LVESD measurements. The left ventricle ejection fraction was measured by the M-mode (LVEF_MMode) at parasternal long axis view using the Teichholz formula. The LVEF_2D was calculated using parasternal short axis view measurements of volumes by the method of area-length. All images were recorded by the same investigator (BPD), experienced in small animals experiments and analyzed offline by three investigators, ALV, BPD, and MMDR, the last one, a specialist in human echocardiography.

\section{Speckle tracking echocardiography}

The global LV myocardium deformation analysis in three axes, longitudinal, circumferential and radial was performed. The global longitudinal strain (GLS) was obtained in a parasternal long axis view, while global circumferential strain (GCS) and radial strain (GRS) were measured in a parasternal short axis view. The twodimensional speckle-tracking analysis was applied to assess LV deformation using the validated $\mathrm{Vevo}^{\circledR}$ Strain Software (VisualSonics Inc., Toronto, Canada). The end-diastole was 
automatically defined at the peak of the QRS complex. The end-systolic time was defined as the point of the smallest dimension of the LV cavity and visually checked by using the position of mitral leaflets in the background image in M20-mode ${ }^{20}$.

The manual LV endocardial border delineation was performed in the parasternal long axis view to measure GLS or short axis view, in order to measure GRS and GCS. Thereafter, epicardial borders were automatically defined, creating a region of interest. If necessary, a manual adjustment in the region of interest was made to include the entire region of $\mathrm{LV}$ myocardium, excluding the pericardium, based on a six-segment model ${ }^{21}$ in each projection. Finally, visual quality control in myocardium tracking was applied, and a retracking was performed when at least one segment was poorly tracked.

In the presence of a persistent poor tracking of at least one segment per projection, the global deformation analysis was considered not feasible. The LV deformation was measured as the peak systolic change in the myocardial length relative to the length at the end of diastole represented by a percentage of change (\%). Negative values represent longitudinal and circumferential myocardial deformation and positive values represent radial deformation.

\section{Reproducibility}

The reproducibility analysis of echocardiographic LVEF and STE parameters was performed in 20 randomly selected animals. For the intra-observer reproducibility, a rereading was carried out at least 60 days after the first reading, blinded to the previous results. For the inter-observer analysis, the second independent reader was blinded to the analysis of the first reader. Inter- and intra-reader reproducibility analyses were evaluated using an intra-class correlation coefficient (ICC) to assess absolute agreement and a coefficient of variation (CV), determined by the standard deviation divided by the mean, and expressed in percentage.

\section{Statistical analysis}

Continuous data presented normal distribution when assessed by histograms and the Shapiro-Wilk test, and are expressed as mean \pm standard deviation (SD). Categorical data are presented as absolute values and percentages. The student t-test was used to evaluate differences between the two groups at baseline and 15 days after the echocardiographic examinations.

The analysis of variance for mixed models of repeated measures (mixed-ANOVA) was used to evaluate interaction (main effect) between the experimental groups (betweensubject effect) and time (within-subject effect). The survival analysis was studied using Kaplan-Meier curves and comparison between groups used the log-rank test. The statistical analysis was performed using Minitab 17 (Minitab Inc. 2010, State College, PA, USA) and Stata 14.0 (StataCorp, College Station, TX, USA), and P-values $<0.05$ were considered statistically significant.

\section{RESULTS}

A total of 45 animals were evaluated by echocardiography. At baseline (Table 1), animals in the CG and IG groups had similar weight but the heart rate of the T. cruzi-infected group was statistically superior to the controls $(184.9 \pm 19.7$ vs $161.4 \pm 26.1 \mathrm{bpm} ; \mathrm{p}=0.009)$. Echocardiographic variables of LV geometry showed a significantly higher value of LVEDD in the chagasic group compared to $\mathrm{CG}(6.5 \pm 0.3$ vs $6.0 \pm 0.5 \mathrm{~mm}$; $\mathrm{p}=0.003)$, but the difference was not present when LVEDD was indexed according to the animals' weight ( $5.04 \pm 0.42$ vs $5.01 \pm 0.42 ; p=0.286)$.

LVEF measured with both methods (MMode and 2D) and FS, LV mass, and STE parameters as GLS and GRS were not different between groups. However, GCS was reduced in the $T$. cruzi-infected group $(-19.5 \pm 3.1 \mathrm{vs}-22.9 \pm 5.6$; $\mathrm{p}=0.0456$ ).

At 15 days after infection, chagasic animals had reduced weight, increased heart rate, and reduced absolute LVEDD, although LVEDD indexed to body weight was increased when compared to the control group. All LVEF and FS measurements decreased in the T. cruzi-infected group, with an elevated LVESD. All STE parameters were reduced in T. cruzi-infected group compared to CG. All data and statistical comparisons are shown in Table 2.

Survival in the $T$. cruzi-infected group was significantly reduced ( $40 \%$ vs $6.6 \% ; \mathrm{p}=0.03$ ) and log-rank comparison of survival Kaplan-Meier curves $(\mathrm{p}=0.02)$. Mortality in the T. cruzi-infected group occurred from day 19 to day 23 post-infection. All the 12 dead animals in the T. cruziinfected group presented with one or more of the clinical signs of disease. The only dead animal in the control group did not present with clinical signs of disease. The survival analysis is shown in Figure 1.

Sequential analysis of LV functional parameters showed that 15 days after the challenge, chagasic animals evolved with reduced LVEF according to all methods with a p-value for interaction equal to 0.003 for LVEF_2D (Figure 2) and a p-value for interaction equal to 0.005 for LVEF_MMode and a p-value for interaction equal to 0.013 for FS. STE parameters analyses showed that GLS was also 
Table 1 - Basal characteristics of animals, the control group vs T. cruzi-infected group.

\begin{tabular}{lccc}
\hline & Control & Chagas & $p$ \\
\hline $\mathrm{n}$ & 15 & 30 & 0.139 \\
Weight (g) & $120.7 \pm 7.9$ & $124.87 \pm 9.95$ & 0.009 \\
HR (bpm) & $161.4 \pm 26.1$ & $184.9 \pm 19.7$ & 0.003 \\
\hline ECHO & & & 0.091 \\
\hline LVEDD (mm) & $6.0 \pm 0.5$ & $6.5 \pm 0.3$ & 0.072 \\
LVESD (mm) & $4.4 \pm 0.5$ & $4.7 \pm 0.3$ & 0.376 \\
LVEF_2D (\%) & $57.9 \pm 7.5$ & $53.8 \pm 4.9$ & 0.485 \\
LVEF_MMode (\%) & $50 \pm 11,1$ & $52.7 \pm 4.9$ & 0.862 \\
FS (\%) & $26.5 \pm 7.0$ & $27.8 \pm 3.2$ & 0.698 \\
LV mass (mg) & $427.9 \pm 77.5$ & $431.9 \pm 56.5$ & 0.286 \\
LV mass/w (g/hg) & $354.02 \pm 58.7$ & $346.89 \pm 45.4$ & 0.456 \\
LVEDD/w (cm/hg) & $5.01 \pm 0.42$ & $5.04 \pm 0.42$ & 0.538 \\
GLS (\%) & $-19.59 \pm 4.0$ & $-18.6 \pm 3.9$ & 0.046 \\
GRS (\%) & $30.19 \pm 8.67$ & $28.54 \pm 6.7$ & \\
GCS(\%) & $-22.9 \pm 5.6$ & $-19.5 \pm 3.1$ & \\
\hline
\end{tabular}

$\mathrm{Bpm}=$ beats per minute; $\mathrm{g}=$ grams $; \mathrm{g} / \mathrm{hg}=$ grams/hectograms; $\mathrm{mm}=$ millimeter $\mathrm{mm} / \mathrm{hg}=$ millimeter per hectagram; $\mathrm{mg}=\mathrm{milligram}$. $\mathrm{HR}=$ heart rate; $\mathrm{LVEDD}=$ left ventricle end-diastolic diameter; $\mathrm{LVESD}=$ left ventricle end systolic diameter; LVEF_2D = left ventricle ejection fraction (2D); LVEF_MMode = left ventricle ejection fraction (MMode); FS = fractional shortening; GLS = global longitudinal strain; GCS = global circumferential strain; GRS = global radial strain.

Table 2 - Characteristics of animals (control group vs T. cruzi-infected group) 15 days post-infection.

\begin{tabular}{lccc}
\hline & Control & Chagas & $p$ \\
\hline $\mathrm{n}$ & 15 & 30 & $<0.05$ \\
Weight (g) & $131.1 \pm 6.7$ & $108.4 \pm 27.4$ & $<0.05$ \\
HR (bpm) & $151.1 \pm 25.2$ & $191.7 \pm 46.5$ & 0.02 \\
Clinical signs (\%) & 0 & 50 & \\
Mortality rate (\%) & 6.6 & 40 & \\
\hline ECHO & & $5.86 \pm 1.1$ & 0.047 \\
\hline LVEDD (mm) & $6.3 \pm 0.3$ & $4.47 \pm 1.1$ & 0.035 \\
LVESD (mm) & $4.2 \pm 0.3$ & $43.6 \pm 12.1$ & $<0.05$ \\
LVEF_2D (\%) & $59.7 \pm 5.9$ & $46.6 \pm 14.6$ & $<0.05$ \\
LVEF_MMode (\%) & $59.3 \pm 8.1$ & $24.6 \pm 10.3$ & $<0.05$ \\
FS (\%) & $32.5 \pm 5.7$ & $526.5 \pm 113.9$ & $<0.05$ \\
LV mass (mg) & $456.2 \pm 59.2$ & $488.1 \pm 81.1$ & $<0.05$ \\
LV mass/w (g/hg) & $348.6 \pm 46.5$ & $5.29 \pm 1.3$ & 0.002 \\
LVEDD/w (mm/hg) & $4.8 \pm 0.3$ & $-13.5 \pm 4.8$ & $<0.05$ \\
GLS (\%) & $-19.7 \pm 4.0$ & $25.5 .1 \pm 7.7$ & $<0.05$ \\
GRS (\%) & $35.1 \pm 10.2$ & $-17.3 \pm 4.7$ & $<0.05$ \\
GCS(\%) & $-21.9 \pm 4.3$ &
\end{tabular}

$\mathrm{bpm}=$ beats per minute; $\mathrm{g}=$ grams; $\mathrm{g} / \mathrm{hg}=$ grams/hectograms; $\mathrm{mm}=$ millimeter $\mathrm{mm} / \mathrm{hg}=$ millimeter per hectagram; $\mathrm{mg}=\mathrm{milligram}$. $\mathrm{HR}=$ heart rate; LVEDD = left ventricle end-diastolic diameter; LVESD = left ventricle end systolic diameter; LVEF_2D = left ventricle ejection fraction (2D); LVEF_Mmode = left ventricle ejection fraction (MMode); FS = fractional shortening; GLS = global longitudinal strain; GCS = global circumferential strain; GRS = global radial strain.

significantly impaired through time in the chagasic group with a p-value for interaction equal to 0.012 (Figure $3 \mathrm{~A}$ ).
Reductions in GCS and GRS through time in the T. cruziinfected group were not statistically significant ( $p$-value for 


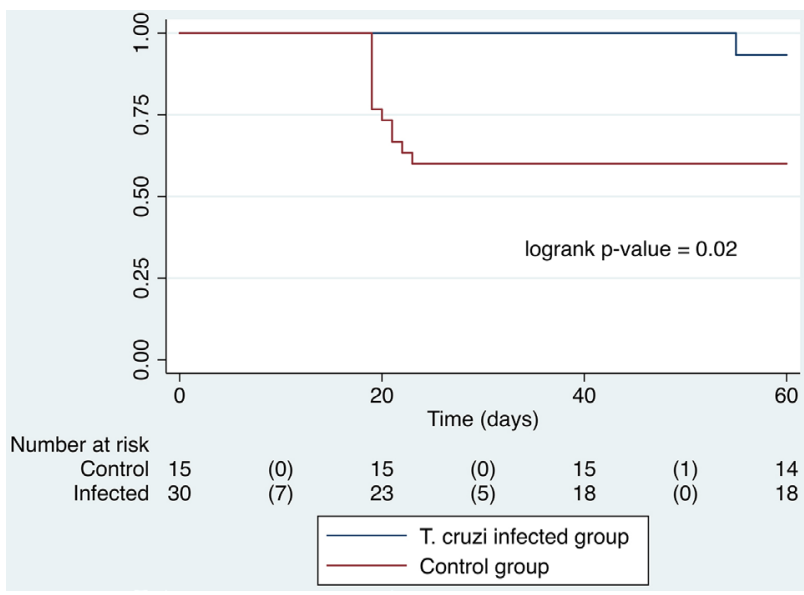

Figure 1 - Survival analysis using Kaplan-Meier curves from both groups (controls and T. cruzi- infected group) during the follow-up.

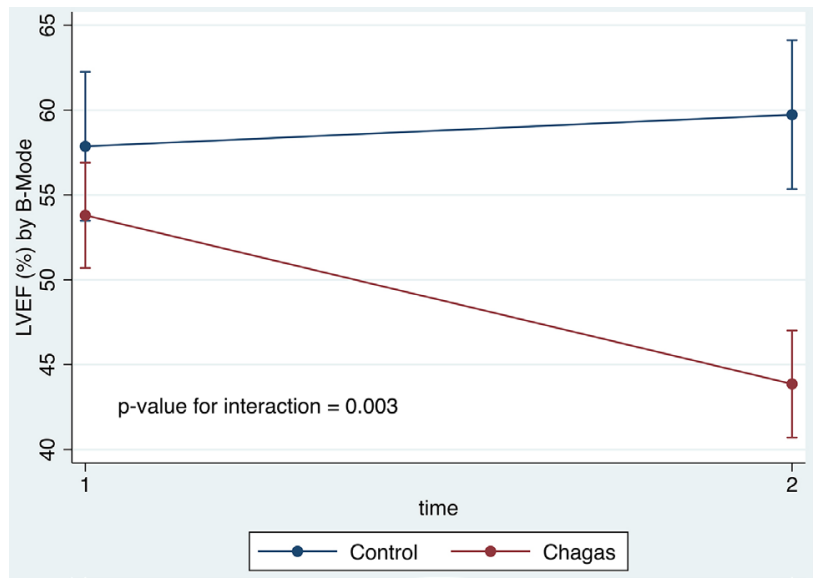

Figure 2 - LVEF_2D evolution through time in controls and $T$. cruzi-infected group.

interaction equal to 0.0493 and 0.069 , respectively) when they were analyzed through ANOVA of mixed models with group\#time interactions (Figures $3 \mathrm{~B}$ and $3 \mathrm{C}$ ).

The reproducibility analysis of LVEF and STE revealed ICC ranging from 0.67 (for GRS) to 0.91 (for GLS). The LVEF_MMode method showed the lowest inter-reader coefficient of variation (5.66\%), while GRS exhibited the highest coefficient $(13.63 \%)$. The intra-reader reproducibility showed an ICC ranging from 0.49 (for LVEF_2D) to 0.92 (for GLS) and a coefficient of variation from $4.25 \%$ (for LVEF_MMode) to $10.85 \%$ (for GRS).

\section{DISCUSSION}

The acute phase of $\mathrm{CD}$ is well reproduced in the Syrian hamster animal model with a high mortality rate in comparison with the control group. Mortality happened from day 19 to day 23 post-infection and was preceded by clinical

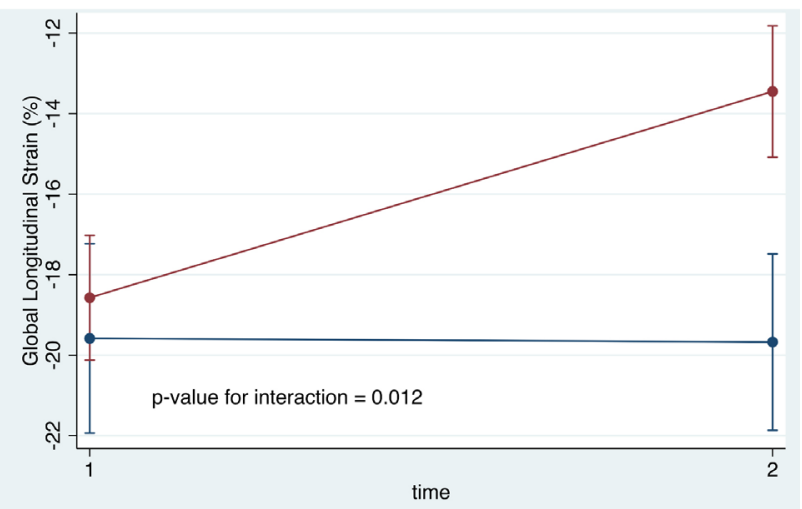

A
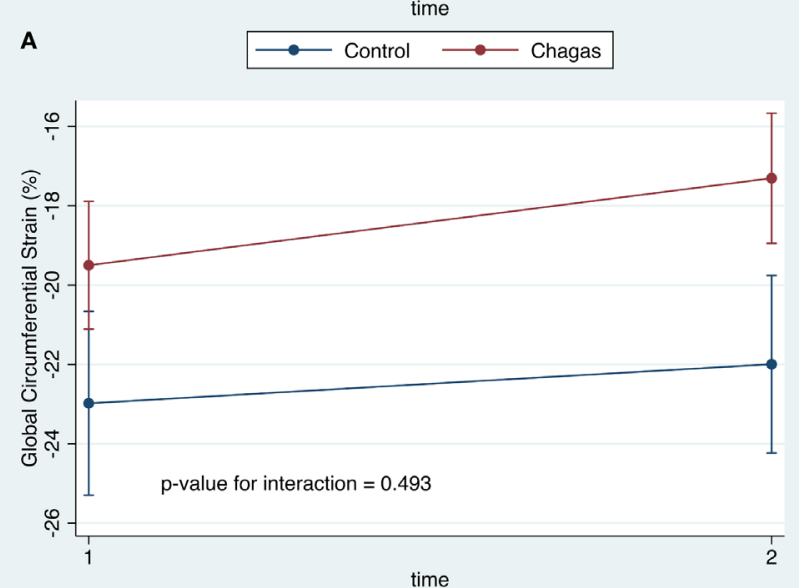

B
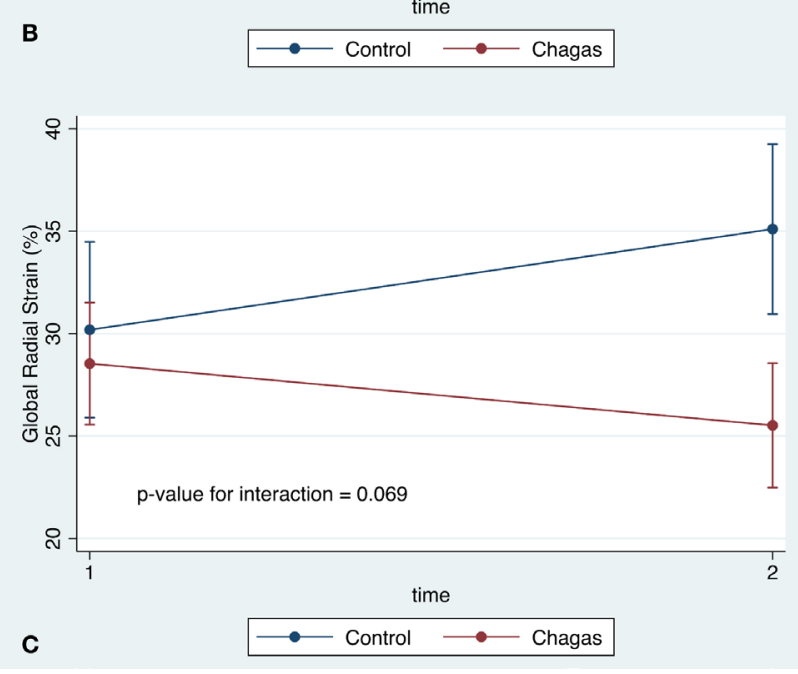

Figure 3 - Myocardial deformation indices: GLS (A), GCS (B) and GRS (C), evolution through time in controls vs $T$. cruziinfected group.

signs in all CD animals. When studied by echocardiography 15 days post-infection, $T$. cruzi-infected animals presented with increased LVEDD/w, increased LVESD and reduced LVEF. Furthermore, the STE parameter of longitudinal deformation, GLS, was reduced at this time of the disease evolution in the T. cruzi-infected group.

Cardiac alterations described in human clinical records of the acute phase of vector-transmitted CD include pericardial 
effusion, LV regional myocardial dysfunction, valvar regurgitations, and, in a proportion of patients, global LV dysfunction, which can be normalized with the etiological treatment ${ }^{8,9,22}$. In the non-vector transmission, such as oral or solid organ transplantation, the LV global dysfunction, presenting as acute myocarditis has been described and clinical evolution can be dramatic ${ }^{6}$. Mortality in the acute phase of human CD is described in 5\% to $10 \%$ of cases $^{22}$. This animal model of CD was already known to reproduce the chronic CD cardiomyopathy, with mortality rates near $50 \%$, at 9 to 11 months of evolution ${ }^{15,23}$. However, this study shows the ability of this model to also reproduce the acute phase of the disease, with left ventricle geometry and systolic function alterations in the first 15 days after infection. Acute myocarditis with $\mathrm{LV}$ dysfunction is responsible for the major proportion of mortality (40\%) through the complete evolution of the disease in the animal model.

Some previous studies with other animal models of $\mathrm{CD}$ failed to demonstrate the presence of consistent left ventricular dysfunction. Lima et al. ${ }^{12}$, studying $T$. cruziinoculated rhesus monkey by means of bidimensional echocardiography, did not show any difference in LVEDD or its area, neither in the systolic change of LV area even in the presence of several degrees of myocarditis in the tissue analysis. This study was conducted in a remote time of the echocardiography evolution, which could explain the lack of sensitivity to evaluate LV geometry and systolic function by the method. Other studies in small animal models as mice have also demonstrated myocarditis in the tissue analysis, but not all of them correlated it to the ventricular function evaluation ${ }^{24}$. Jeliks et al. ${ }^{25}$, however, using gated magnetic cardiac resonance in a mice model of CD, demonstrated that LVEDD, as well as RVEDD, increased in acute $\mathrm{CD}$, although these authors have not yet reported data of ventricular function. After this, the same group demonstrated a reduced LV fractional shortening (FS) in this rodent animal model of acute $\mathrm{CD}$, which could be used as a parameter of response to treatments ${ }^{26}$. Left ventricle FS was, since then, used in other animal studies as an echocardiography variable capable to demonstrate systolic LV dysfunction in acute CD but there are still some controversies ${ }^{23,27}$. Difficulties may be related to the fact that FS is generated from a unidimensional linear LV measurement and it is not able to represent segmental LV myocardial hypokinesia. The use of bidimensional methods to measure LVEF has been recently applied to small animal models of cardiac disease, following the development of a dedicated equipment with high-resolution transducers ${ }^{17,28}$. Our data showed a consistently reduced LV systolic function in the first 15 days of infection, measured by three different echocardiographic methods: FS, LVEF from MMode and the bidimensional mode. In addition, a significant geometrical remodeling is demonstrated in this animal model in the acute phase of CD, with increments of LVmass, LVEDD/w and LVESD. Thus, an acute dilated cardiomyopathy has been well demonstrated in the acute phase of CD in the Syrian hamster animal model with this study.

The left ventricle deformation analysis in the acute phase of CD in our study was significantly reduced. GLS was statistically reduced in chagasic animals compared to controls 15 days after challenge, however, GCS and GRS reduction trends were not statistically significant. Statistical differences noted in simple group comparisons were not confirmed when using the mixed-ANOVA model, which adjusts exposition to infection and time of evolution together. The GLS behavior is not well known through the acute phase of $\mathrm{CD}$. Previous studies in humans showed that, through the evolution of $\mathrm{CD}$, individuals in the indeterminate phase of the disease do not present with a significant reduction of GLS ${ }^{18,19,29}$, but no data about LV deformation indexes were demonstrated in the acute phase. As mortality happens in the acute phase, it is plausible that survivals from the acute phase undergoing the chronic phase, even if presenting with indeterminate forms of $\mathrm{CD}$, are those who do not have dysfunction in the acute phase. GLS is known as a sensitive parameter of myocardial dysfunction that can be depressed in the early phases of other non-ischemic myocardial diseases. However, considering its reduction in the acute phase, together with other LV geometric and classic functional parameters as LVEF, it does not represent early cardiac damage, but an overt expressed acute myocarditis. The influence of the heart rate in the echocardiographic analysis of myocardium deformation parameters is always a technical concern. We have previously published data showing little influence of the heart rate in the longitudinal myocardial deformation analysis in normal Hamsters, using the same equipment ${ }^{20}$. Although animals were not infected in the previous publication, the heart rate range was $204 \pm 24 \mathrm{bpm}$, and it was even superior in comparison with the T. cruzi-infected group of the present study. The same previous study showed that the animals weigh had no significant influence in myocardial deformation parameters.

The measurement of GRS has yielded more controversial data in studies on patients with diverse forms of chronic $\mathrm{CD}^{18,29}$. Although chronic Chagas disease presents typical myocardial damage of meso-myocardium fibrosis ${ }^{30}$, presuming that GCS and GRS should be more compromised, once this myocardium deformation direction represents essentially the meso-myocardial fiber function, our study showed that these parameters are still not significantly altered 
in acute CD. High variability of GRS is still a recognized problem of the technique that can be explained by several factors, such as image formation and tracking of speckles ${ }^{31}$. However, we should also consider inflammation as a more prominent mechanism of acute $\mathrm{CD}^{32}$ than myocardial fibrosis, and also question if GCS and GRS can demonstrate it.

\section{Limitations}

Our findings should be interpreted in the context of several limitations. At first, the fact that only animals who had all echocardiographic analyses in both time points were included, according to the inherent characteristic of the mixed-ANOVA analysis. Secondly, this study did not present the tissue analysis, as deaths were spontaneous and the short time interval of the acute phase did not allow euthanasia of the very sick animals. Finally, it is also important to consider that anesthetic agents could influence parameters such as the myocardial performance. Although isoflurane is considered by some authors as a more superficial anesthetic agent, it has significant effects on cardiac function evaluated by $\mathrm{LVEF}^{33}$. Animals in this study used ketamine based on the fact that it has been shown to have fewer effects on LVEF and that it has been already tested in hamsters ${ }^{34}$. Nevertheless, there is a lack of data in the literature about the impact of anesthetic agents in myocardial deformation parameters.

\section{Translational aspects and future perspectives}

In acute $\mathrm{CD}$, early and sequential monitoring of myocardial function with a non-invasive method as echocardiography allows the detection of LV geometrical changes as LVEDD/w and LVmass/w increases and also a reduction in LVEF. The myocardial deformation analysis in the longitudinal axis (GLS) can be also detected. Geometrical and functional compromise precedes high mortality in acute $\mathrm{CD}$ and can be used as a prognostic function marker. A major proportion of dead animals present, before death, clinical signs of disease. Considering a proportionally significant non-vector transmission of CD, acute cases are still prevalent and if they are symptomatic, they should have cardiac function and geometry strictly monitored.

\section{CONCLUSION}

Mortality in the acute phase of CD in the Syrian hamster animal model is high and timely related to the myocardial systolic dysfunction. Dysfunction can be detected with conventional echocardiography techniques such as LVEF quantification, and also with STE parameters as a reduction in the longitudinal deformation, measured by GLS.

\section{AUTHORS' CONTRIBUTIONS}

Conception and design of the study, or acquisition of data, or analysis and interpretation of data: ALV, BPD, HTM, ECOF, DMT, MMDR; drafting the article or revising it critically for important intellectual content: BPD, AS, BCM, MVS, JAMN, MMDR. Final approval of the version to be submitted: MMDR.

\section{CONFLICT OF INTERESTS}

The authors declare no conflict of interests.

\section{FUNDING}

This work was supported by the Fundação de Apoio à Pesquisa do Estado de São Paulo (FAPESP), Brazil, grant $\mathrm{N}^{\circ}$ 2016/25403-9, and Conselho Nacional de Desenvolvimento Científico e Tecnológico (CNPq), Brazil, grant $\mathrm{N}^{\circ} 132280 / 2019-1$.

\section{REFERENCES}

1. Santos EF, Silva AA, Leony LM, Freitas NE, Daltro RT, RegisSilva CG, et al. Acute Chagas disease in Brazil from 2001 to 2018: a nationwide spatiotemporal analysis. PLoS Negl Trop Dis. 2020;14:e0008445.

2. Prata A. Clinical and epidemiological aspects of Chagas disease. Lancet Infect Dis. 2001;1:92-100.

3. Perez-Molina JA, Molina I. Chagas disease. Lancet. 2018;391:8294.

4. Requena-Méndez A, Aldasoro E, de Lazzari E, Sicuri E, Brown M, Moore DA, et al. Prevalence of Chagas disease in LatinAmerican migrants living in Europe: a systematic review and meta-analysis. PLoS Negl Trop Dis. 2015;9:e0003540.

5. Dias JC. Human chagas disease and migration in the context of globalization: some particular aspects. J Trop Med. 2013;2013:789758

6. Souza FF, Castro ES, Marin Neto JA, Sankarankutty AK, Teixeira AC, Martinelli AL, et al. Acute chagasic myocardiopathy after orthotopic liver transplantation with donor and recipient serologically negative for Trypanosoma cruzi: a case report. Transplant Proc. 2008;40:875-8.

7. Chin-Hong PV, Schwartz BS, Bern C, Montgomery SP, Kontak $\mathrm{S}$, Kubak B, et al. Screening and treatment of chagas disease in organ transplant recipients in the United States: recommendations from the chagas in transplant working group. Am J Transplant. 2011;11:672-80. 
8. Dias JC, Ramos Jr AN, Gontijo ED, Luquetti A, Shikanai-Yasuda MA, Coura JR, et al. 2nd Brazilian Consensus on Chagas Disease, 2015. Rev Soc Bras Med Trop. 2016;49 Suppl 1:360.

9. Bastos CJ, Aras R, Mota G, Reis F, Dias JP, de Jesus RS, et al. Clinical outcomes of thirteen patients with acute chagas disease acquired through oral transmission from two urban outbreaks in northeastern Brazil. PLoS Negl Trop Dis. 2010;4:e711.

10. Shikanai-Yasuda MA, Lopes MH, Tolezano JE, Umezawa E, Amato Neto V, Barreto AC, et al. Doença de Chagas aguda: vias de transmissão, aspectos clínicos e resposta à terapêutica específica em casos diagnosticados em um centro urbano. Rev Inst Med Trop Sao Paulo. 1990;32:16-27.

11. Parada H, Carrasco HA, Anez N, Fuenmayor C, Inglessis I. Cardiac involvement is a constant finding in acute Chagas' disease: a clinical, parasitological and histopathological study. Int J Cardiol. 1997;60:49-54.

12. Lima JA, Szarfman A, Lima SD, Adams RJ, Russell RJ, Cheever A, et al. Absence of left ventricular dysfunction during acute chagasic myocarditis in the rhesus monkey. Circulation. 1986;73:172-9.

13. Jelicks LA, Tanowitz HB. Advances in imaging of animal models of Chagas disease. Adv Parasitol. 2011;75:193-208.

14. Bilate AM, Salemi VM, Ramires FJ, De Brito T, Silva AM, Umezawa ES, et al. The Syrian hamster as a model for the dilated cardiomyopathy of Chagas' disease: a quantitative echocardiographical and histopathological analysis. Microbes Infect. 2003;5:1116-24.

15. Oliveira LF, Romano MM, Carvalho EE, Cabeza JM, Salgado HC, Fazan Junior R, et al. Histopathological correlates of global and segmental left ventricular systolic dysfunction in experimental chronic Chagas cardiomyopathy. J Am Heart Assoc. 2016;5:e002786.

16. Schmidt A, Romano MM, Marin-Neto JA, Rao-Melacini P, Rassi Jr A, Mattos A, et al. Effects of trypanocidal treatment on echocardiographic parameters in Chagas cardiomyopathy and prognostic value of Wall Motion Score Index: a BENEFIT trial echocardiographic substudy. J Am Soc Echocardiogr. 2018;32:286-95.e3.

17. Ram R, Mickelsen DM, Theodoropoulos C, Blaxall BC. New approaches in small animal echocardiography: imaging the sounds of silence. Am J Physiol Heart Circ Physiol. 2011;301:H1765-80.

18. Romano MM, Moreira HT, Marin-Neto JA, Baccelli PE, Alenezi F, Klem I, et al. Early impairment of myocardial deformation assessed by regional speckle-tracking echocardiography in the indeterminate form of Chagas disease without fibrosis detected by cardiac magnetic resonance. PLoS Negl Trop Dis. 2020;14:e0008795.

19. Gomes VA, Alves GF, Hadlich M, Azevedo CF, Pereira IM, Santos CR, et al. Analysis of regional left ventricular strain in patients with Chagas disease and normal left ventricular systolic function. J Am Soc Echocardiogr. 2016;29:67988

20. Barros Filho AC, Moreira HT, Dias BP, Ribeiro FF, Tanaka DM, Schmidt A, et al. Feasibility and reference intervals assessed by conventional and speckle-tracking echocardiography in normal hamsters. Physiol Rep. 2021;9:e14776.

21. Morgan EE, Faulx MD, McElfresh TA, Kung TA, Zawaneh MS, Stanley WC, et al. Validation of echocardiographic methods for assessing left ventricular dysfunction in rats with myocardial infarction. Am J Physiol Heart Circ Physiol. 2004;287:H204953.

22. Punukollu G, Gowda RM, Khan IA, Navarro VS, Vasavada BC. Clinical aspects of the Chagas' heart disease. Int J Cardiol. 2007;115:279-83.

23. Pimentel WS, Ramires FJ, Lanni BM, Salemi VM, Bilate AM, Cunha-Neto E, et al. The effect of beta-blockade on myocardial remodelling in Chagas' cardiomyopathy. Clinics (Sao Paulo). 2012;67:1063-9.

24. Leon JS, Wang K, Engman DM. Captopril ameliorates myocarditis in acute experimental Chagas disease. Circulation. 2003;107:2264-9.

25. Jelicks LA, Shirani J, Wittner M, Chandra M, Weiss LM, Factor $\mathrm{SM}$, et al. Application of cardiac gated magnetic resonance imaging in murine Chagas' disease. Am J Trop Med Hyg. 1999;61:207-14.

26. Jelicks LA, Chandra M, Shirani J, Shtutin V, Tang B, Christ GJ, et al. Cardioprotective effects of phosphoramidon on myocardial structure and function in murine Chagas' disease. Int J Parasitol. 2002;32:1497-506.

27. De Souza AP, Tanowitz HB, Chandra M, Shtutin V, Weiss LM, Morris SA, et al. Effects of early and late verapamil administration on the development of cardiomyopathy in experimental chronic Trypanosoma cruzi (Brazil strain) infection. Parasitol Res. 2004;92:496-501.

28. Esper L, Roman-Campos D, Lara A, Brant F, Castro LL, Barroso A, et al. Role of SOCS2 in modulating heart damage and function in a murine model of acute Chagas disease. Am J Pathol. 2012;181:130-40.

29. García-Álvarez A, Sitges M, Regueiro A, Poyatos S, Jesus Pinazo M, Posada E, et al. Myocardial deformation analysis in Chagas heart disease with the use of speckle tracking echocardiography. J Card Fail. 2011;17:1028-34.

30. Volpe GJ, Moreira HT, Trad HS, Wu KC, Braggion-Santos MF, Santos MK, et al. Left ventricular scar and prognosis in chronic Chagas cardiomyopathy. J Am Coll Cardiol. 2018;72:2567-76.

31. Voigt JU, Pedrizzetti G, Lysyansky P, Marwick TH, Houle H, Baumann R, et al. Definitions for a common standard for 2D speckle tracking echocardiography: consensus document of the EACVI/ASE/Industry Task Force to standardize deformation imaging. J Am Soc Echocardiogr. 2015;28:183-93. 
32. Carneiro AC, Costa GP, Ferreira CS, Ramos IP, Sarandy MM, Leite AL, et al. Combination therapy with benznidazole and doxycycline shows no additive effect to monotherapy with benznidazole in mice infected with the VL-10 strain of the Trypanosoma cruzi. Int J Cardiol. 2020;299:243-8.

33. Pachon RE, Scharf BA, Vatner DE, Vatner SF. Best anesthetics for assessing left ventricular systolic function by echocardiography in mice. Am J Physiol Heart Circ Physiol. 2015;308:H1525-9.

34. Tanaka DM, Romano MM, Carvalho EE, Oliveira LF, Souza HC, Maciel BC, et al. Effect of different anesthetic agents on left ventricular systolic function assessed by echocardiography in hamsters. Braz J Med Biol Res. 2016;49:e5294. 